\title{
Sollten Pflegeroboter auch sexuelle Assistenzfunktionen bieten?
}

\author{
Nicola Döring
}

\section{Zusammenfassung}

Pflege und Pflegeroboter zielen darauf ab, Menschen mit Pflegebedarf zu betreuen, Krankheiten zu verhüten und Gesundheit zu fördern. Das umfasst die ganzheitliche Sorge um das Wohlbefinden und schließt auch das sexuelle Wohlbefinden als wichtigen Gesundheitsfaktor und als Menschenrecht mit ein. Der Beitrag behandelt zunächst den Stellenwert des sexuellen Wohlbefindens und konkretisiert die sexuellen Anliegen von Menschen mit Pflegebedarf. Die bisherigen Angebote der Sexualassistenz für Menschen mit Pflegebedarf werden beschrieben. Auf dieser Grundlage plädiert der Beitrag dafür, im Sinne einer besseren Förderung sexuellen Wohlbefindens von Menschen mit Pflegebedarf in Zukunft auch Konzepte der robotischen Sexualassistenz zu entwickeln und zu evaluieren. Dabei werden drei Entwicklungspfade aufgezeigt, nämlich die Nutzung von Sexrobotern und Sexpuppen, die Ausstattung von multifunktionalen Pflegerobotern mit sexuellen Assistenzfunktionen sowie der Einsatz spezialisierter sexueller Assistenzgeräte. In allen drei Szenarien sollte robotische Sexualassistenz jeweils durch professionelle Sexualberatung begleitet werden. Angesichts der Tatsache, dass in der Allgemeinbevölkerung das Interesse an und die Nutzung von sexuellen Artefakten wie Sexspielzeugen und Sexmaschinen weit verbreitet und positiv mit sexueller Gesundheit verknüpft ist, sind Menschen mit Pflegebedarf von dieser Entwicklung nicht auszuschließen. Möglichen Gefahren robotischer Sexualassistenz gilt es im Zuge einer interdisziplinär informierten Entwicklung der Technik und ihrer Einsatzszenarien entgegenzuwirken.

\footnotetext{
N. Döring $(\square)$

Institute of Media and Communication Science IfMK, Ilmenau University of Technology, Ilmenau, Deutschland E-Mail: Nicola.Doering@tu-ilmenau.de

(C) Der/die Autor(en) 2018 


\subsection{Einleitung}

Unter Gesundheits- und Pflegerobotern (health care robots, care robots) verstehen wir jene Service- und Assistenzroboter, die der Gesundheits- und Pflegeunterstützung dienen (zur Klassifikation unterschiedlicher Roboterarten siehe Siciliano und Kathib 2016). Die Forschung und Entwicklung im Bereich der Pflegeroboter verläuft in zwei Richtungen:

- Zum einen werden Pflegeroboter zur Unterstützung des Pflegepersonals in stationären Einrichtungen entwickelt (z. B. Heberoboter RIBA II von Riken). Hier geht es darum, die Arbeitsbedingungen der Pflegekräfte zu verbessern, sie idealerweise von körperlich anstrengenden Routineaufgaben zu entlasten (z. B. Hilfe beim Heben und Umbetten von Bewohner_innen, beim Transport von Wäsche und Mahlzeiten) und ihnen damit mehr Freiräume für menschliche Zuwendung zu eröffnen (BGW 2017).

- Zum anderen werden aber auch Gesundheits- und Pflegeroboter entwickelt, die das selbstständige Leben von Menschen mit Pflege- und Assistenzbedarf im häuslichen Umfeld unterstützen (z. B. Care-O-bot vom Fraunhofer-Institut für Produktionstechnik und Automatisierung; Twendy-One von der Waseda University). Diese persönlichen Assistenzroboter sollen typischerweise bestimmte Aufgaben im Haushalt übernehmen, Sicherheitsfunktionen bieten, die Mobilität erleichtern sowie der sozialen, geistigen und körperlichen Aktivierung und Gesundheitspflege dienen (z. B. Hilfe beim Toilettengang, Anleitung von Reha-Gymnastik, Erinnerung an regelmäßiges Trinken, Überwachung der Medikamenteneinnahme). Oberstes Ziel ist es dabei, Menschen mit Pflege- und Assistenzbedarf mehr Selbstständigkeit zu ermöglichen und ihre Lebensqualität einschließlich Gesundheit und Wohlbefinden zu steigern oder zumindest zu erhalten (Döring et al. 2015; Vandemeulebroucke et al. 2017).

Ob und wie diese hochgesteckten Ziele in der Praxis tatsächlich erreicht werden können, inwiefern Pflegeroboter von Menschen mit Pflegebedarf, von Pflegenden, Pflegeeinrichtungen sowie deren Trägern akzeptiert werden und welche Gefahren Pflegeroboter mit sich bringen - all dies ist seit rund zehn Jahren Gegenstand empirischer Forschung und kontroverser ethischer Debatten.

Interessanterweise wurden im Kontext der Entwicklung von Gesundheits- und Pflegerobotern Fragen des sexuellen Wohlbefindens und der sexuellen Assistenz bislang noch nicht vertieft behandelt (Bendel 2015). Dabei gibt es mindestens zwei gute Gründe, um der Frage nachzugehen, ob Pflegeroboter nicht auch sexuelle Assistenzfunktionen übernehmen sollten:

1. In Forschung und Praxis wird in den letzten Jahren immer weniger ignoriert, dass Menschen mit Pflege- und Assistenzbedarf (also z. B. ältere Menschen mit wachsenden gesundheitlichen Einschränkungen, aber auch jüngere Menschen mit unterschiedlichen körperlichen, seelischen und geistigen Behinderungen) durchaus sexuelle Bedürfnisse haben und artikulieren, diese jedoch aus verschiedenen Gründen bislang 
ungenügend ausleben können (Mahieu et al. 2011; Mahieu und Gastmans 2015; Palacios-Cena et al. 2016; Shakespeare et al. 1997). Das gilt für das häusliche Umfeld ebenso wie für stationäre Einrichtungen wie z. B. Wohn- und Pflegeheime. Könnten hier Pflegeroboter durch sexuelle Assistenzfunktionen - zumindest für bestimmte Zielgruppen - hilfreiche und willkommene Lösungen anbieten?

2. Parallel zur Entwicklung von Pflegerobotern wird in den letzten Jahren die Entwicklung von Sexrobotern vorangetrieben (Döring 2017b; Levy 2007; Sharkey et al. 2017). Aktuelle Prognosen gehen davon aus, dass es bereits im Jahr 2050 für Frauen wie Männer völlig normal sein wird, mit Robotern Sex zu haben, und zwar befriedigenden Sex (Levy 2007; Pearson 2016). Die ersten Sexroboter sind bereits erhältlich. Auch der Markt für Vorläuferformen dieser neuen Technik boomt: Lebensechte Sexpuppen werden inzwischen in diversen Formen und Preisklassen vermarktet und auch bereits von nennenswert großen Bevölkerungsgruppen genutzt: In einer repräsentativen Online-Umfrage in Deutschland gaben $2 \%$ der Frauen und $9 \%$ der Männer Erfahrungen mit Sexpuppen an (Döring und Pöschl, 2018). Welche Potenziale Sexpuppen und Sexroboter für die altersbedingt beständig wachsende Gruppe von Menschen mit Pflege- und Assistenzbedarf bieten könnten, wurde bislang nicht systematisch betrachtet. Hier wäre dann auch zu klären, ob und wenn ja welche Menschen mit Pflegebedarf einen zusätzlichen Sexroboter, einen Pflegeroboter mit sexuellen Assistenzfunktionen oder eher spezialisierte sexuelle Assistenzgeräte bevorzugen.

Der vorliegende Beitrag befasst sich erstmals mit der Frage, ob und wie die sexuellen Anliegen von Menschen mit Pflegebedarf - zumindest zum Teil - durch robotische Assistenz bedient werden könnten und sollten. Er setzt dazu direkt beim sexuellen Wohlbefinden an und rekonstruiert dieses zunächst als empirisch nachgewiesenen Gesundheitsfaktor und als international verbrieftes Menschenrecht (Abschn. 14.2). Anschließend wird dargestellt, was wir über die sexuellen Anliegen speziell von Menschen mit Pflege- und Assistenzbedarf wissen (Abschn. 14.3). Um diesen Anliegen gerecht zu werden, wird bereits heute sexuelle Assistenz durch menschliche Fachkräfte angeboten (Abschn. 14.4). Vor dem Hintergrund dieser bisherigen Formen sexueller Assistenz behandelt der Beitrag dann die sexuelle Assistenz durch Roboter und andere Assistenzgeräte (Abschn. 14.5). Die Bestandsaufnahme endet mit einem Fazit für die zukünftige Forschung und Entwicklung (Abschn. 14.6).

\subsection{Sexuelles Wohlbefinden als Gesundheitsfaktor und Menschenrecht}

Wenn Pflegeroboter dazu beitragen sollen, die Lebensqualität von Menschen mit Pflegebedarf zu steigern oder zumindest zu erhalten, dann darf das sexuelle Wohlbefinden dabei nicht ausgeblendet werden. Denn nach heutigem Diskussionsstand ist sexuelles Wohlbefinden als Gesundheitsfaktor und Menschenrecht anzuerkennen. 


\subsubsection{Sexuelles Wohlbefinden als Gesundheitsfaktor}

Die Weltgesundheitsorganisation definiert schon lange, dass Gesundheit mehr ist als die Abwesenheit von Krankheit, nämlich umfassendes physisches und psychisches Wohlbefinden beinhaltet (WHO 1946). Dasselbe gilt für sexuelle Gesundheit (sexual health). Sie ist mehr als die Abwesenheit von sexuell übertragbaren Infektionen, sexuellen Funktionsstörungen, ungeplanten Schwangerschaften und sexueller Gewalt - sie schließt das sexuelle Wohlbefinden (sexual well-being) ausdrücklich ein (WHO 2006).

Die WHO-Definitionen von Gesundheit bzw. sexueller Gesundheit haben einen normativen Charakter und geben vor, dass neben der Bekämpfung von Krankheiten eben weltweit auch eine staatliche Gesundheitsförderung stattfinden soll, die das Wohlbefinden der Bevölkerung stärkt. So gibt denn auch beispielsweise das staatliche Gesundheitsportal von Großbritannien (www.nhs.uk) ausdrücklich „good sex tips“ und propagiert offiziell sexuelle Aktivitäten zur Gesundheitsförderung, denn ,sex is good for your heart".

Tatsächlich mehren sich in der medizinischen Forschung die Evidenzen, dass sexuelles Wohlbefinden gesundheitsförderlich ist. Sexuelles Wohlbefinden im Sinne selbst gewählter und subjektiv positiv erlebter sexueller Aktivität trägt kausal über unterschiedliche Mechanismen (z. B. kardiovaskulärer Trainingseffekt, Testosteronproduktion, Stressreduktion, Schmerzreduktion, partnerschaftliche Bindung) zur psychischen und physischen Gesamtgesundheit bei. Dabei gibt es empirische Nachweise zu positiven Gesundheitseffekten sowohl von Solosexualität (Coleman 2003; Levin 2007; Robbins et al. 2011) als auch von Partnersexualität (Levin 2007; Brody 2010; Liu et al. 2016).

Sexuelle Aktivität in gesundheitlichen Kategorien zu diskutieren bringt den Vorteil, dass das ehemals tabubehaftete Feld des Sexuellen entstigmatisiert und besser besprechbar wird. Gleichzeitig können aber auch im Vokabular des Gesundheitsdiskurses weiterhin sexualmoralische Vorgaben transportiert werden. Was früher ,unsittlich“ war, kann heute als ,,ungesund“ ausgegrenzt werden. Sexualität ist und bleibt ein Feld der Moralpolitik.

Ebenso bewegen wir uns immer in einem Spannungsverhältnis zwischen neuen Freiheiten und neuen Einschränkungen: Der moderne „Sex-ist-gesund“-Diskurs kann neuen Formen von Verunsicherung, Leistungsdruck, Selbstoptimierung und Kommerzialisierung Vorschub leisten (Döring 2017a). Die wachsende Nachfrage nach luststeigernden Präparaten für Mann und Frau mag ein Beispiel dafür sein, dass die frühere scham- und schuldbehaftete Sorge, zu viel Sex zu haben oder zu wollen, mittlerweile der angstvollen Sorge gewichen ist, zu wenig oder zu wenig lustvollen Sex zu haben. Der „Sex-ist-gesund“Diskurs ist also auch kritisch zu sehen, wenn er neue Handlungszwänge konstruiert und z. B. Menschen mit geringer sexueller Motivation oder asexueller Orientierung ausgrenzt (Gupta 2011). Gleichwohl trägt der ,Sex-ist-gesund“-Diskurs dazu bei, die sexuellen Anliegen aller Menschen - einschließlich derjenigen mit Pflegebedarf - ernster zu nehmen. Ein anderer Bedeutungsrahmen, der im Pflegekontext gern verwendet wird, beschreibt Sexualität als Lebensenergie und Lebendigkeit und spricht neben der Gesundheit somit auch spirituelle Aspekte an (BTT-Gruppe 2016; Döring 2017a). 


\subsubsection{Sexuelles Wohlbefinden als Menschenrecht}

Dass Pflegeroboter durchaus auch für sexuelle Assistenz zuständig sein könnten und sollten, ist nicht nur aus medizinischen und spirituellen Gründen („Sex ist gesund“; „Sex ist positive Lebensenergie“") bedenkenswert, sondern auch aus ethischen Erwägungen. So wird sexuelle Gesundheit einschließlich sexuellem Wohlbefinden heute international als sexuelles Menschenrecht anerkannt.

Diverse politisch agierende Institutionen wie die UN (United Nations), die WHO (World Health Organization), die IPPF (International Planned Parenthood Federation) und die WAS (World Association for Sexual Health) haben im Zuge internationaler Expertenkonsultationen Deklarationen sexueller und reproduktiver Menschenrechte entwickelt und veröffentlicht. Die WAS beispielsweise hat ihre „Declaration of Sexual Rights“ (WAS 2014) in den letzten 20 Jahren bereits vier Mal überarbeitet, was auf einen anhaltend lebendigen Diskurs in diesem Feld hindeutet.

Allgemein besteht Einigkeit darüber, dass sexuelle Menschenrechte in zwei Gruppen einzuteilen sind:

- Schutzrechte (auch: negative sexuelle Menschenrechte) beziehen sich darauf, Menschen vor einer Beeinträchtigung ihrer sexuellen Gesundheit und ihres sexuellen Wohlbefindens zu schützen (z. B. Schutz vor sexueller Gewalt, Schutz vor Zwangsehe, Schutz vor sexuell übertragbaren Infektionen).

- Freiheitsrechte (auch: positive sexuelle Menschenrechte) beziehen sich darauf, Menschen den Freiraum zum Ausdrücken und Ausleben der eigenen Sexualitäten - ohne Schaden für andere - zu ermöglichen (z. B. Recht auf Sexualaufklärung, Freiheit zu eigener Partnerwahl und Familienplanung).

Empirische Forschung zielt darauf ab zu erfassen, welche sexuellen Menschenrechte in unterschiedlichen Ländern und/oder bei unterschiedlichen Bevölkerungsgruppen verletzt oder geachtet werden (Glasier et al. 2006). Nach wie vor wird das Konstrukt der sexuellen Menschenrechte aber auch kritisch diskutiert, etwa hinsichtlich seiner historischen Entwicklung und ungelösten Fragen (Lottes 2013; Tiefer 2002). Wenn jeder Mensch ein Recht auf sexuelles Wohlbefinden hat und der Staat hier fördernd eingreifen soll, aber nur begrenzte Ressourcen verfügbar sind, wer erhält dann eine Förderung und wer nicht? Der junge Mann mit Muskellähmung ebenso wie der alte Mann im Frühstadium einer Demenz, der Mann mit massiven Schüchternheitsproblemen ebenso wie die Frau mit sexueller Traumatisierung, die Witwe, die körperliche Nähe vermisst, aber ihrem verstorbenen Mann treu bleiben will, ebenso wie der Witwer, den es ins Rotlichtmilieu zieht? Durch Ressourcenknappheit entsteht bei Unterstützungsbedarf nicht nur eine Konkurrenz zwischen verschiedenen Anspruchsgruppen, sondern auch zwischen verschiedenen Bedürfnissen: Wenn die angesichts Pflegenotstand ohnehin überlasteten Pflegekräfte ihre letzten verfügbaren Ressourcen in die Förderung sexuellen Wohlbefindens investieren, welche anderen Bedürfnisse der Menschen mit Pflegebedarf bleiben dafür auf der Strecke? 
Jene Forschungsfelder, die sich interdisziplinär mit Behinderung (Disability Studies) und mit dem Altern (Aging Studies) befassen, behandeln sexuelle Fragen typischerweise in einem rechtebasierten Paradigma. Dabei wird kritisiert, dass Menschen mit Behinderung (Shakespeare et al. 1997; Shakespeare 2000; Foley 2018) ebenso wie ältere Menschen (Barrett und Hinchliff 2018; Brähler und Berberich 2008; Grond 2011; Van der Vight-Klußmann 2014) oft per se als asexuell angesehen werden und strukturell in ihren sexuellen Selbstbestimmungsrechten beschnitten werden, etwa wenn in der Altenund Behindertenpflege, aber auch im häuslichen Umfeld keine passenden Möglichkeiten zum Ausleben von Solo- und Partnersexualität geschaffen werden. Daraus leitet sich die Forderung ab, das Freiheitsrecht auf sexuellen Selbstausdruck bei Menschen mit Pflegebedarf stärker anzuerkennen, Angehörige und Pflegepersonal sexualpädagogisch, sexualmedizinisch und sexualpsychologisch weiterzubilden sowie im häuslichen wie stationären Umfeld entsprechend sexualfreundliche Rahmenbedingungen zu schaffen.

Gleichzeitig wird aber auch davor gewarnt, das Recht auf sexuellen Selbstausdruck ungewollt zur neuen Pflicht zu erklären und insbesondere die Vielfalt der sexuellen Bedürfnisse zu übersehen. Ältere Menschen oder Menschen mit Behinderung an allzu engen und für sie unpassenden Normvorstellungen über „richtigen“ oder ,gesunden“ Sex zu messen (z. B. „Sex ist heterosexueller Geschlechtsverkehr“), widerspricht eben genau der Vorstellung von sexueller Selbstbestimmung.

\subsection{Sexuelle Anliegen von Menschen mit Pflege- und Assistenzbedarf}

Wenn die bisherige Forschung sich mit dem Sexualverhalten und den sexuellen Anliegen von Menschen mit Behinderungen oder altersbedingten Einschränkungen beschäftigt hat, dann lag der Fokus oft eher auf den Schutzrechten als auf den Freiheitsrechten.

\subsubsection{Schutz vor Gewalt und reproduktive Rechte}

Schutz vor sexueller Gewalt ist ein zentrales sexuelles Menschenrecht, das im Pflegekontext in zweifacher Weise besonders virulent ist:

- Schutz vor Viktimisierung durch sexuelle Gewalt. Es ist gut belegt, dass Mädchen und Frauen mit Behinderung in besonders starkem Maße dem Risiko sexueller Viktimisierung ausgesetzt sind, und dass bei älteren Frauen sexuelle Viktimisierung häufiger übersehen wird, weshalb hier Schutzkonzepte sehr wichtig sind (Brownridge 2006; Wacker et al. 2009; Plummer und Findley 2012; Jones et al. 2012; BMFSFJ 2013).

- Prävention von sexuell unangemessenem und grenzverletzendem Verhalten. Menschen mit kognitiven Beeinträchtigungen, etwa aufgrund von Demenzerkrankungen oder 
Lernbehinderungen, fallen immer wieder durch sexuell unangemessenes und grenzverletzendes Verhalten gegenüber Mitbewohner_innen und Pflegenden auf, sodass hier Präventionsmaßnahmen eine wichtige Rolle spielen (Lindsay 2002; Wilson und Burns 2011).

Im Hinblick auf das Freiheitsrecht der selbstbestimmten Familienplanung ist zu beachten, dass Menschen mit geistiger und/oder körperlicher Behinderung nicht selten einen Kinderwunsch haben. Dieser kann z. B. im Zuge begleiteter Elternschaft erfüllt werden (Tilley et al. 2012; BMFSFJ 2013).

\subsubsection{Freiheit zu sexuellem Selbstausdruck}

Hinsichtlich der Freiheit zu sexuellem Selbstausdruck und sexuellem Wohlbefinden bestehen bei Menschen mit Pflegebedarf große Forschungslücken (McCabe und Taleporos 2003; Tepper 2000; Shakespeare 2000). Einige der wenigen Fachbeiträge, die sich der Frage widmen, wie Menschen mit Pflege- und Assistenzbedarf erwünschte sexuelle Aktivitäten erleben können und welche Rahmenbedingungen sie dafür benötigen, seien im Folgenden herausgegriffen.

Der Ratgeber „Supporting Disabled People with their Sexual Lifes“ von Tuppy Owens (2015) basiert auf der rund 40-jährigen Erfahrung der Autorin mit der Organisation von „Outsiders“, einer britischen Kontaktbörse für Menschen mit Behinderung. Ihrer Beobachtung nach haben Menschen mit Behinderung vor allem vier zentrale Anliegen bezüglich ihres Sexuallebens:

1. Sie möchten sich sexuell frei fühlen.

2. Sie wünschen sich sexuelle Aufklärung.

3. Sie erwarten Unterstützung im Falle von sexuellen Negativerfahrungen und Krisen.

4. Sie möchten sexuelle Aktivitäten genießen.

In der Fachliteratur wird erörtert, dass Angehörige und Pflegende Menschen mit Beeinträchtigungen oft vor Enttäuschungen in der Liebe und Sexualität bewahren möchten (Foley 2018), dass zu viel Schutz aber eben Entfaltungsmöglichkeiten beschneide und es auch ein „Recht auf Liebeskummer“ gebe (Sandfort 2010). Zum Genuss solound partnersexueller Aktivitäten sind laut Owens (2015) aus Sicht von Menschen mit Behinderung vor allem folgende sieben Punkte ausschlaggebend:

1. Nutzung von Pornografie

2. Nutzung von Sexspielzeug

3. Umarmungen

4. Zusammen in einem Bett schlafen 
5. Partnersexualität

6. Nutzung von sexuellen Dienstleistungen

7. Finanzielle Mittel für Sexspielzeug und sexuelle Dienstleistungen

Die Bedürfnisse von älteren und auch sehr alten Menschen werden ähnlich beschrieben (Brähler und Berberich 2008; Grond 2011). Insbesondere ist die Vorstellung unzutreffend, dass ältere Menschen weitgehend asexuell leben. Umfragen zeigen, dass neben der Solosexualität mehr als die Hälfte der 70-Jährigen (Beckmann et al. 2008) und knapp ein Drittel der 77-Jährigen Partnersexualität praktizieren (Matthias et al. 1997). Auch Hochbetagte mit Demenzerkrankungen artikulieren sexuelle Wünsche (Sramek 2015), wobei diese bei stark fortgeschrittener Erkrankung zurücktreten (Mück 2013). Die sexuellen Selbstbestimmungsrechte der Bewohner_innen stellen Pflegeeinrichtungen vor die Herausforderung, entsprechende Rahmenbedingungen zu schaffen (Grond 2011; Van der Vight-Klußmann 2014), etwa genügend Privatsphäre durch von innen abschließbare Zimmer, die Akzeptanz von romantischen und sexuellen Paarbeziehungen zwischen Bewohner_innen oder auch die Bereitstellung von Pflegedoppelbetten.

Betrachtet man aktuelle Leitlinien zum Umgang mit Sexualität in Einrichtungen der Seniorenpflege, so zeigt sich eine wachsende Sensibilität für die sexuellen Selbstbestimmungsrechte der Menschen mit Pflegebedarf - auch in kirchlichen Einrichtungen (z. B. BTT-Gruppe 2016). Gleichzeitig werden aber auch die Grenzen deutlich, etwa wenn festgelegt ist, dass das Pflegepersonal professionelle Distanz zu wahren habe, und nicht nur keine sexuellen Kontakte zu Bewohner_innen aufnehmen darf, sondern ihnen auch keine Pornografie und Sexspielzeuge beschaffen oder sexuellen Dienstleistungen organisieren darf (BTT-Gruppe 2016). Bei der Bestimmung und Aushandlung derartiger Grenzen geht es neben den sexuellen Freiheitsrechten der pflegebedürftigen Personen gleichzeitig immer auch um die Selbstbestimmungsrechte und Grenzen der (mehrheitlich weiblichen) Pflegefachkräfte, denen nicht automatisch zugemutet werden darf, sich ständig mit den sexuellen Belangen der Bewohner und Bewohnerinnen befassen zu müssen.

Das Ausleben von Sexualität in Pflegeeinrichtungen ist nicht zuletzt auch durch die Werte und Normen der Mitbewohner_innen reglementiert, die generationsbedingt z. B. homosexuelle Beziehungen teilweise offen ablehnen. Hier ist die Pflegeeinrichtung gefragt, ein wertschätzendes Klima für sexuelle Vielfalt zu schaffen (BTT-Gruppe 2016). Es existieren inzwischen auch spezielle Einrichtungen der „Regenbogenpflege“ (Marquardt 2017). Erst vereinzelt werden im Kontext der Pflege jetzt auch Transidentität und Intergeschlechtlichkeit thematisiert. Dabei ist ein Verständnis für die Vielfalt von Geschlechtsidentitäten wichtig, damit trans* oder inter* Bewohner_innen im Sinne sexueller Menschenrechte keiner Diskriminierung durch Mitbewohner_innen und/oder Pflegepersonal ausgesetzt sind. Vor besonderen Herausforderungen stehen schließlich informell Pflegende, etwa wenn sie mit der tabuisierten Sexualität ihrer pflegebedürftigen Eltern oder mit der veränderten Sexualität ihrer pflegebedürftigen Lebenspartner_innen konfrontiert sind (Reichert und Karrasch 2013; Mück 2013). Unwissenheit und fehlende Kommunikation erschweren hier oft einen lustvollen Umgang mit Sexualität oder verhindern ihn gänzlich. 


\subsection{Sexuelle Assistenz durch menschliche Fachkräfte}

Wenn wir davon ausgehen, dass zumindest ein Teil der Menschen mit Pflegebedarf sexuelle Anliegen haben und ihre sexuellen Selbstbestimmungsrechte verwirklichen wollen, dafür aber sowohl in stationären Einrichtungen als auch im häuslichen Umfeld Unterstützung benötigen, stellt sich die Frage nach der sexuellen Assistenz. Unter sexueller Assistenz bzw. Sexualassistenz (sexual assistance) werden unterstützende sexuelle Dienstleistungen für Menschen zusammengefasst, die aufgrund ihrer Behinderung oder altersbedingter Einschränkungen ihre Sexualität nicht ohne Unterstützung leben können (Senf 2013). Der Fachdiskurs unterscheidet Sexualberatung als passive Sexualassistenz von Sexualbegleitung als aktiver Sexualassistenz (pro familia 2005, S. 6). Zudem nehmen Menschen mit Pflegebedarf aber auch herkömmliche sexuelle Dienstleistungen in Anspruch.

\subsubsection{Sexualberatung}

Sexualberatung im Sinne passiver Sexualassistenz schafft Bedingungen und Voraussetzungen für sexuelle Aktivitäten, ohne dass die Fachkraft selbst aktiv in sexuelle Handlungen involviert wird. Im Rahmen von passiver Sexualassistenz können die Klient_innen z. B. unterstützt werden, indem sie bislang fehlende Sexualaufklärung bekommen (BZgA 2015) und/oder indem sie beratende und organisatorische Hilfe bei der Beschaffung von Pornografie und Sexspielzeug, bei der Partnersuche oder beim Zugang zu sexuellen Dienstleistungen erhalten. Passive Sexualassistenz kann etwa beinhalten, zusammen mit einer Person mit geistigen oder körperlichen Einschränkungen geeignete Sexspielzeuge zu suchen und einen geschützten Raum zur ungestörten Nutzung zu schaffen, oder Zugang zu einer sexuellen Dienstleistung (Bordellbesuch, Empfang eines Escorts) zu organisieren. Das Angebotsspektrum passiver Sexualassistenz variiert - nicht zuletzt da die damit verbundenen ethischen Fragen kontrovers diskutiert werden (z. B. wird Unterstützung bei Bordellbesuchen teilweise abgelehnt). Passive Sexualassistenz wird in Deutschland beispielsweise von pro familia angeboten, etwa in Form von aufsuchender Sexualberatung in Pflegeeinrichtungen (www.profamilia.de/themen/sexualitaet-und-behinderung.html). Passive Sexualassistenz erfreut sich wachsender Akzeptanz.

\subsubsection{Sexualbegleitung}

Sexualbegleitung im Sinne aktiver Sexualassistenz ermöglicht sinnlich-sexuelle Nähe mit der Fachkraft, die oft als Sexualbegleiter_in bezeichnet wird und als Surrogatpartner_in fungiert. Der Kontakt zwischen Sexualbegleiter_innen und ihren Klient_innen konzentriert sich auf Umarmungen, Streicheln, Nacktheit, Massage und unterstützte Selbstbefriedigung. Oft ist Geschlechtsverkehr ausgeschlossen. Sexualassistenz grenzt 
sich somit von Prostitution als kommerziellem Sex ab und betont stärker die Fürsorge für beeinträchtigte Einzelpersonen sowie die Unterstützung für beeinträchtigte Paare. Dienstleistende kommen oft aus den Bereichen Pflege, Sozialpädagogik und Tantra und müssen in Pflegeeinrichtungen Externe sein, denn aus ethischen und rechtlichen Gründen darf kein Abhängigkeitsverhältnis vorliegen. Trotzdem besteht bei aktiver Sexualassistenz ein erhöhtes Risiko sexueller Viktimisierung der pflegebedürftigen Personen. Das gilt insbesondere für Frauen mit Behinderung oder Beeinträchtigungen.

Für aktive Sexualassistenz werden Stundensätze von 100 bis 150 EUR abgerufen, was über den Preisen für herkömmliche sexuelle Dienstleistungen liegt. Hinzu kommen oft noch die Kosten für lange Anfahrtswege. Das Angebot an aktiver Sexualassistenz ist in Deutschland und auch europaweit bislang noch sehr begrenzt und in vielen Regionen gar nicht vor Ort verfügbar (pro familia 2005). In Tab. 14.1 findet sich eine Auswahl an Angeboten der aktiven Sexualassistenz, wobei die Selbstbeschreibungen der Sexualbegleiter_innen auf ihren jeweiligen Internetpräsenzen das fachliche Selbstverständnis verdeutlichen (siehe ergänzend YouTube-Videos mit Edith Arnold, Stephanie Klee oder Milka Reich). Die Angebote zielen meist auf Menschen mit Behinderungen und altersbedingten Beeinträchtigungen, zum Teil auch auf schüchterne und liebesunerfahrene Personen.

\subsubsection{Sexarbeit}

Nicht zuletzt aufgrund der hohen Kosten für Sexualbegleitung und des sehr begrenzten Angebots wird für Menschen mit Pflegebedarf auch herkömmliche Sexarbeit als Alternative zu den für sie oft schwer anzubahnenden privaten Sexualbeziehungen genutzt. Die Erotikbranche stellt sich auf die wachsende Zahl von älteren Menschen und Menschen mit Pflegebedarf ein: Zunehmend mehr Bordelle werben mit Barrierefreiheit, zunehmend mehr Sexarbeiter_innen spezialisieren sich auf diese Klientel. Auch wird vonseiten der Menschen mit Beeinträchtigungen teilweise ausdrücklich herkömmliche Sexarbeit der Sexualbegleitung vorgezogen, da neben Massagen eben auch Geschlechtsverkehr gewünscht wird. Ein Medienbericht begleitete den Inklusionsaktivisten Klaus Birnstiehl beim Bordellbesuch, den er der aktiven Sexualassistenz vorzieht, und zitiert ihn mit der Aussage „Ich will nicht nur kuscheln“ (Mattner 2015). In seinem Inklusionsblog beschreibt der Rollstuhlfahrer „Rolliman“ seine positiven Erfahrungen im Rotlichtmilieu (www.rollimans-erfahrungen.de). Hauptsächlich nachgefragt werden sexuelle Körperkontaktdienstleistungen in Form herkömmlicher Sexarbeit oder Sexualbegleitung bislang von heterosexuellen Männern mit Behinderung oder altersbedingten Beeinträchtigungen, wobei die Kosten in der Regel selbst getragen werden müssen und nicht von Krankenkassen oder Sozialversicherungen übernommen werden (pro familia 2005). 


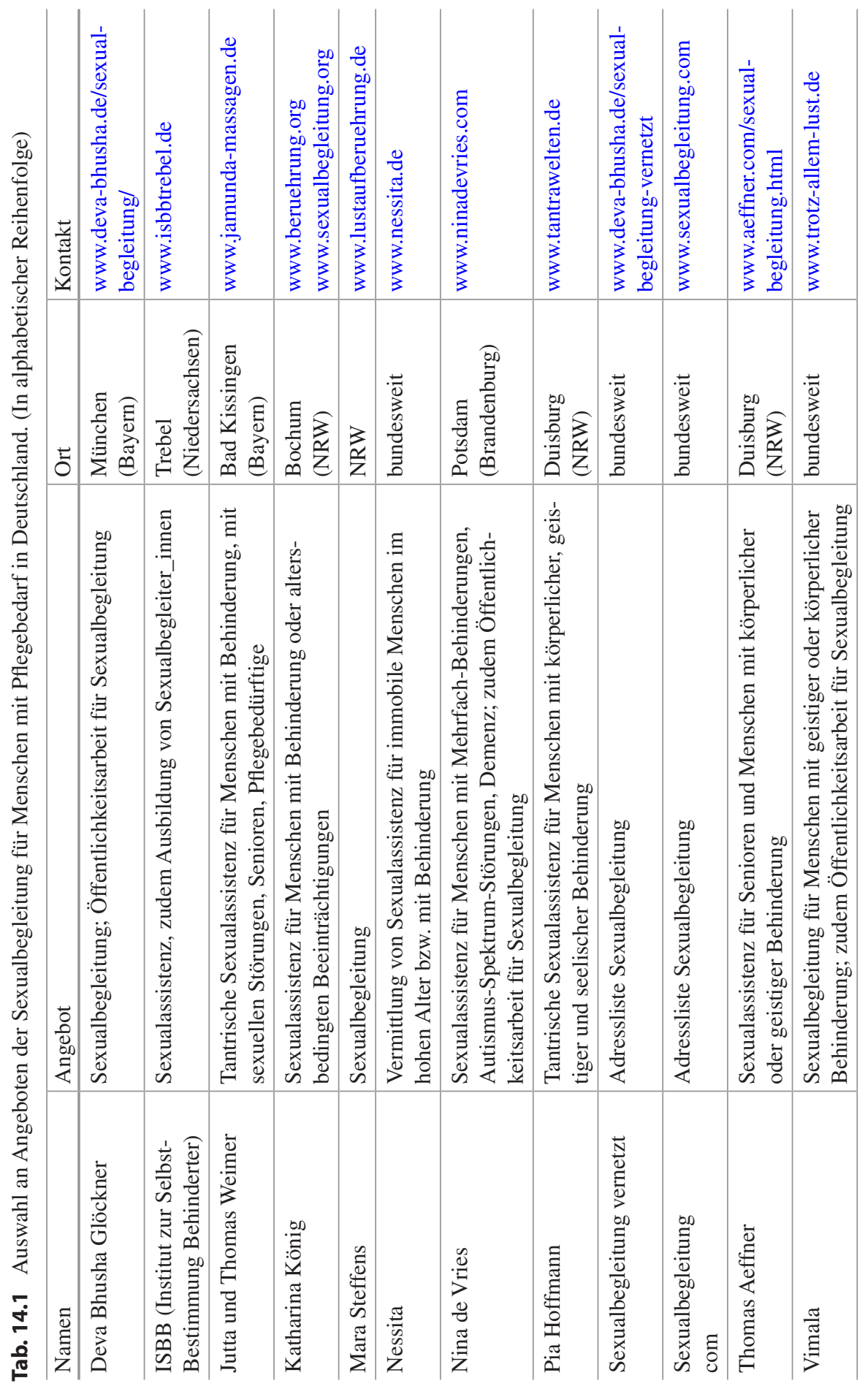




\subsection{Sexuelle Assistenz durch Roboter und andere Assistenzgeräte}

Will man für die sexuellen Anliegen von Menschen mit Pflegebedarf auch technische Lösungen in Erwägung ziehen, so sind drei Ansätze zu diskutieren, und zwar die Nutzung von Sexrobotern und Sexpuppen, der Einsatz von Pflegerobotern mit sexuellen Assistenzfunktionen sowie der Gebrauch von spezialisierten sexuellen Assistenzgeräten.

\subsubsection{Sexroboter und Sexpuppen}

Stellt man die heute einschlägigen Pflegeroboter (z. B. Care-O-bot oder Twendy-One) den heute verfügbaren Sexrobotern (z. B. Roxxxy oder Rocky von TrueCompanion.com; Samantha von SyntheaAmatus.com) gegenüber, so fallen deutliche Unterschiede auf. Während die Pflegeroboter meist ein technisch-funktionales Aussehen haben, um Aufgaben im Haushalt und in der Pflege zu bewältigen, sind die Sexroboter so menschenähnlich wie möglich gestaltet - einschließlich funktionaler Genitalien. Dementsprechend könnte man von einer strikten Aufgabenteilung der Roboter ausgehen. Ebenso wie Menschen ohne Pflegebedarf könnten Menschen mit Pflegebedarf neben ihrem Pflegeroboter potenziell auch einen Sexroboter nutzen, sofern sie ihn denn finanzieren können. Sexroboter könnten zur Förderung des sexuellen Wohlbefindens eingesetzt werden, insbesondere, wenn aufgrund von körperlichen und/oder geistigen Beeinträchtigungen das Anknüpfen sexueller Beziehungen zu anderen Menschen erschwert und möglicherweise auch Solosexualität nicht eigenhändig möglich ist.

Um die Interaktivität und künstliche Intelligenz zukünftiger Sexroboter optimal zu nutzen, wären Modelle zu entwickeln, die sich auf bestimmte körperliche oder geistige Beeinträchtigungen spezialisieren und durchaus auch Trainingsprogramme beinhalten. Denkbar wären Sexroboter, mit denen bei sexuellen Funktionsstörungen angst- und schamfrei geübt werden kann, ebenso wie Sexroboter, mit denen bei sexueller Traumatisierung ein sicherer und kontrollierter Kontakt erlebt werden kann. Ein Ausschöpfen dieser Potenziale würde es erfordern, dass sich Roboter-, Sexual-, Alterns- und Pflegeforschung interdisziplinär zusammenschließen und unter Einbeziehung der Zielgruppen Konzepte der robotischen Assistenz für sexuelles Wohlbefinden und sexuelle Gesundheit entwickeln und evaluieren (Döring 2017b). Angesichts der hohen Kosten von Sexrobotern ist individueller Besitz bis auf Weiteres für Menschen mit Pflegebedarf meist keine Option. Denkbar sind Dienstleistungsangebote, die Sexroboter stundenweise vermieten und dabei durch spezialisierte Roboter und zielgruppengerechte Beratung auch Menschen mit Behinderung und Beeinträchtigungen ansprechen.

Für bestimmte Zielgruppen, die ungedeckten Bedarf nach Nähe, Körperkontakt und Sexualität haben, mag anstelle eines technisch anspruchsvollen und kostenintensiven Sexroboters schon eine Sex- bzw. Liebespuppe ausreichen. So berichten Puppenbesitzer in öffentlichen Online-Foren über den therapeutischen Nutzen der Puppen, die Einsamkeit 
reduzieren, bei der Tagesstrukturierung helfen, beim Einschlafen beruhigend und tröstend wirken und auch sexuell befriedigen können (Döring 2017b; Döring und Pöschl 2018). Liebespuppen haben bislang noch ein negatives Image, könnten aber in Zukunft stärker normalisiert werden. Wenn wir akzeptieren, dass der Umgang mit einem Robotertier (wie etwa der Roboterrobbe Paro: www.parorobots.com) emotional hilfreich auf pflegebedürftige Menschen wirkt und dort sinnvoll ist, wo keine realen Haustiere gehalten werden können, warum soll dann nicht eine sexuelle Assistenzpuppe dort genutzt werden, wo aktive Sexualassistenz mangels Angebot oder Finanzierungsmöglichkeiten nicht zur Verfügung steht? Erste Sexpuppenbordelle existieren in Deutschland bereits (z. B. www. bordoll.de in Dortmund).

Es bestehen große ethische und psychologische Bedenken, die emotionale Bindung pflegebedürftiger Menschen an Robotertiere oder Robotermenschen zu fördern - sei es, weil Pflegebedürftige den Artefaktcharakter der Roboter vielleicht nicht richtig verstehen und/oder weil der soziale Kontakt zu tierischen oder menschlichen Lebewesen eben im Sinne von Humanität und Wohlbefinden nicht durch parasoziale Kontakte zu robotischen Pseudolebewesen verdrängt werden soll. Doch von Verdrängung kann im Falle von Liebes- und Sexualpartner_innen oft keine Rede sein, da sehr viele Menschen mit Pflegebedarf solche Kontakte eben über Jahre hinweg gar nicht haben und auch kaum aufbauen können. Erklärungsbedürftig ist auch, warum wir Erwachsenen den tröstlichen Umgang mit einer Liebespuppe oder einem Liebesroboter nicht zutrauen und zugestehen wollen, während es kulturell weithin akzeptiert ist, dass Kinder ihre Puppen lieben, mit ihnen kuscheln, sprechen, einschlafen und sie als Gefährten behandeln (Döring 2017b).

\subsubsection{Pflegeroboter mit sexuellen Assistenzfunktionen}

Anstelle eines zusätzlichen Liebes- oder Sexroboters ist auch zu überlegen, ob und wie man einen Pflegeroboter um sexuelle Assistenzfunktionen ergänzen kann. Bei Bewegungseinschränkungen (z. B. nach einem Schlaganfall) kann ein Pflegeroboter wie der Care-O-bot oder der Twendy-One mit einem Roboterarm etwa auch dazu genutzt werden, ein Sexspielzeug anzureichen, zu positionieren und zu halten. Mit solchen robotischen Handgriffen wäre die Person mit Pflegebedarf unabhängiger im Ausleben ihrer Solosexualität und müsste nicht Angehörige oder Pflegepersonal um Hilfe bei dieser intimen Angelegenheit bitten. Der Pflegeroboter könnte auch diskret die Reinigung des Sexspielzeugs übernehmen, eine wichtige Aufgabe, für die sich im Pflegealltag bislang kaum jemand zuständig fühlt.

Bei der Konzeption von Pflegerobotern sexuelle Assistenzfunktionen von Anfang an mitzudenken und zusammen mit den Zielgruppen zu entwickeln, erfordert eine Orientierung aller Beteiligten an den Paradigmen der sexuellen Gesundheit und der sexuellen Menschenrechte. Andernfalls wird das Thema Sexualität stigmatisiert und marginalisiert bleiben. 


\subsubsection{Spezialisierte sexuelle Assistenzgeräte}

Für bestimmte sexuelle Anliegen von Menschen mit Pflegebedarf mag es gar nicht notwendig oder sinnvoll sein, einen kompletten Roboter zu nutzen. Hier können kleinere und handlichere Assistenzgeräte im Sinne spezialisierter Sexspielzeuge sinnvoller sein. Sie sind zudem oft diskreter. Einzelgeräte werden auch in anderen Bereichen der robotischen Assistenz eingesetzt. So fungiert etwa ein robotischer Löffel als Mahlzeitenassistent (,My Spoon“: www.secom.co.jp/english/myspoon/).

Weitgehend unter dem Radar der Forschung hat sich der Markt an Sextoys, die von Frauen und Männern für Solo- und Partnersexualität verwendet werden, in den letzten Jahren sehr stark diversifiziert und auch technisiert (Döring 2017b; Döring und Pöschl 2018). Kleinstbetriebe fertigen „Sexmaschinen“, die sich eigenständig bewegen, oder maßgeschneiderte „Sexmöbel“ und vermarkten sie erfolgreich online. Die Entwicklung innovativer Sexprodukte wie des bionischen Umschnalldildos Ambrosia Vibe (Orgasmatronics.com) wird über Crowdfunding-Plattformen wie Indiegogo.com finanziert. Derartige Sexprodukte können besonders hilfreich sein für Menschen mit altersbedingten Beeinträchtigungen, da sie z. B. sexuelle Funktionsstörungen ausgleichen, Mobilität unterstützen und Stimulation verstärken. Die wenigen Studien zu Gebrauch und Wirkung von Sexspielzeugen in der Allgemeinbevölkerung deuten auf positive Gesundheitseffekte hin (Döring und Pöschl 2018).

Somit sind zum einen Initiativen zu fördern, die Sexspielzeuge entwickeln, welche auf die vielfältigen Bedürfnisse von Menschen mit Pflegebedarf zugeschnitten sind (Alptraum 2015). Zum anderen sollte im Rahmen passiver Sexualassistenz für Menschen mit Pflegebedarf bei Bedarf eine zeitgemäße Beratung zu Auswahl, Beschaffung und Nutzung sexueller Assistenzgeräte stattfinden. Denn der Markt ist unübersichtlich und dynamisch. Die Erotikbranche stellt sich langsam auf die Zielgruppe der älteren Menschen ein und vertreibt zunehmend „Produkte der sexuellen Gesundheit“, die etwa sexuellen Lustgewinn und gezieltes Beckenbodentraining zur Inkontinenzprophylaxe kombinieren. Sowohl Inklusionsaktivistinnen wie Tuppy Owens (2014) als auch Sexbloggerinnen wie Erika Lynae (2016) liefern erste Hinweise darauf, welche Sextoys für Menschen mit Beeinträchtigungen besonders nützlich sind. Hier besteht anhaltender Aufklärungs- und Forschungsbedarf.

\subsection{Fazit}

Wenn wir sexuelles Wohlbefinden als Gesundheitsfaktor und Menschenrecht von pflegebedürftigen Personen ernst nehmen, liegt es auf der Hand, im Kontext der Entwicklung von Pflegerobotern auch über robotische Sexualassistenz nachzudenken. Dabei sind sowohl Konzepte denkbar, die sexuelle Assistenzfunktionen in multifunktionale Pflegeroboter integrieren, als auch Konzepte, die mit separaten Sexrobotern oder sexuellen Assistenzgeräten operieren. Sozio-emotional sinnvolle und das Wohlbefinden fördernde Assistenzkonzepte müssen in interdisziplinären Teams zusammen mit den möglichen zukünftigen 
Nutzenden und ihren Angehörigen bzw. gesetzlichen Betreuer_innen sowie den Pflegekräften, Pflegeeinrichtungen und deren Trägern entwickelt werden. Wichtig ist es dabei im Sinne sexueller Freiheitsrechte, die Vielfalt der sexuellen Anliegen von Menschen mit Pflegebedarf zu berücksichtigen und nicht vorschnell sexualmoralisch zu begrenzen. Besonderes Augenmerk ist darauf zu legen, dass die sexuellen Anliegen von Frauen erstmals umfassend erkundet werden und dann auch Berücksichtigung finden (Döring 2017b). Denn geschlechtsrollen- und generationsbedingt sind die heutigen Frauen mit Pflegebedarf oft noch äußerst zurückhaltend darin, sexuelle Bedürfnisse zu artikulieren, und oft werden sie ihnen gesellschaftlich auch gar nicht zugestanden (Barrett und Hinchcliff 2018).

Neben Unterstützung bei der Ausübung sexueller Freiheitsrechte gilt es natürlich, die sexuellen Schutzrechte zu wahren. So muss robotische Sexualassistenz sicher sein. Das betrifft den Schutz sensibler Daten über sexuelle Präferenzen und Gewohnheiten, die bei der Nutzung robotischer Sexualassistenz anfallen, aber auch die Verhinderung von Verletzungen und Unfällen beim Technikgebrauch.

Nicht zuletzt erfordert sexuelle Teilhabe für Menschen mit Pflegebedarf angesichts zunehmender Technisierung, Medikalisierung und Kommerzialisierung des Sexuellen (Döring 2017a) entsprechende finanzielle Mittel, die im Sozialgesetzbuch nicht vorgesehen sind (Zinsmeister 2017). Die Tendenz geht auch eher dahin, die Kostenübernahme für Maßnahmen, die allein der Steigerung der Lebensqualität einschließlich sexuellen Wohlbefindens dienen, weiter abzubauen (so werden z. B. Kosten für Medikamente zur Behandlung erektiler Dysfunktion von den gesetzlichen und privaten Kassen inzwischen nicht mehr übernommen). Von der wachsenden Anerkennung von Sexualität als Gesundheitsfaktor und Menschenrecht können Menschen mit Pflegebedarf aber nur dann profitieren, wenn die notwendigen Rahmenbedingungen auch finanzierbar sind.

Abschließend ist nicht zu vergessen, neben (roboter-)technischen Lösungen weiterhin an nichttechnischen Lösungen für verbessertes sexuelles Wohlbefinden zu arbeiten, etwa durch ausreichende Sexualberatung und vermehrte zwischenmenschliche Kontaktmöglichkeiten.

\section{Literatur}

Alptraum, L. (2015). How to design sex toys for people with disabilities. Motherboard. https:// motherboard.vice.com/en_us/article/9a3987/how-to-design-sex-toys-for-people-with-disabilities. Zugegriffen: 1. März 2018.

Barrett, C., \& Hinchcliff, S. (2018). Addressing the sexual rights of older people: Theory, policy and practice. London: Routledge.

Beckmann, N., Waern, M., Gustafson, D., \& Skoog, I. (2008). Secular trends in self-reported sexual activity and satisfaction in Swedish 70 year olds: Cross sectional survey of four populations, 1971-2001. BMJ, 337, a279.

Bendel, O. (2015). Surgical, Therapeutic, Nursing and Sex Robots in Machine and Information Ethics. In S. P. van Rysewyk \& M. Pontier (Hrsg.), Machine medical ethics. Series: Intelligent systems, control and automation: Science and engineering (S. 17-32). Berlin: Springer. 
Berufsgenossenschaft für Gesundheitsdienst und Wohlfahrtspflege - BGW. (2017). Pflege 4.0 - Einsatz moderner Technologien aus der Sicht professionell Pflegender. Forschungsbericht. https:// www.bgw-online.de/DE/Medien-Service/Medien-Center/Medientypen/BGW-Broschueren/ BGW09-14-002-Pflege-4-0-Einsatz-moderner-Technologien.html. Zugegriffen: 1. März 2018.

Brähler, E., \& Berberich, H. J. (2008). Sexualität und Partnerschaft im Alter. Gießen: Psychosozial Verlag.

Brody, S. (2010). The relative health benefits of different sexual activities. The Journal of Sexual Medicine, 7(4), 1336-1361. doi: https://doi.org/10.1111/j.1743-6109.2009.01677.x.

Brownridge, D. A. (2006). Partner violence against women with disabilities: Prevalence, risk, and explanations. Violence Against Women, 12(9), 805-822. doi: https://doi. org/10.1177/1077801206292681.

BTT-Gruppe. (2016). Umgang mit Sexualität in den Einrichtungen der Seniorendienste. Leitlinie. https://www.bbtgruppe.de/media/docs/broschueren/Umgang-mit-Sexualitaet-Einrichtungen-SD_BBT-Leitlinie.pdf. Zugegriffen: 1. März 2018.

Bundesministerium für Familie, Senioren, Frauen und Jugend - BMFSFJ. (2013). Lebenssituationen und Belastungen von Frauen mit Behinderungen und Beeinträchtigungen in Deutschland. Endbericht. https://www.bmfsfj.de/blob/94206/1d3b0c4c545bfb04e28c$1378141 \mathrm{db} 65 \mathrm{a} /$ lebenssituation-und-belastungen-von-frauen-mit-behinderungen-langfassung-ergebnisse-der-quantitativen-befragung-data.pdf. Zugegriffen: 1. März 2018.

Bundeszentrale für gesundheitliche Aufklärung - BZgA. (2015). Sexualaufklärung für Menschen mit Beeinträchtigungen. Konzept. https://publikationen.sexualaufklaerung.de/index.php?docid=3482. Zugegriffen: 1. März 2018.

Coleman, E. (2003). Masturbation as a means of achieving sexual health. Journal of Psychology \& Human Sexuality, 14, 5-16. doi: https://doi.org/10.1300/J056v14n02_02.

Döring, N. (2017a). Männliche Sexualität im Digitalzeitalter: Aktuelle Diskurse, Trends und Daten. In Stiftung Männergesundheit (Hrsg.), Dritter Deutscher Männergesundheitsbericht (S. 39-75). Gießen: Psychosozial Verlag.

Döring, N. (2017b). Vom Internetsex zum Robotersex. Forschungsstand und Herausforderungen für die Sexualwissenschaft. Zeitschrift für Sexualforschung, 30(1), 35-57.

Döring, N., \& Pöschl, S. (2018). Sex toys, sex dolls, sex robots - Our under-researched bedfollows. Sexologies, 27(3), e51-e55. doi: https://doi.org/10.1016/j.sexol.2018.05.009.

Döring, N., Richter, K., Gross, H.-M., Schröter, C., Müller, S., Volkhardt, M., Scheidig, A., \& Debes, K. (2015). Robotic companions for older people: A case study in the wild. Studies in Health Technology and Informatics, 219, 147-152.

Foley, S. (2018). Intellectual disability and the right to a sexual life: A continuation of the autonomy/paternalism debate. London: Routledge.

Glasier, A., Gülmezoglu, A. M., Schmid, G. P., Moreno, C. G., \& Look, P. F. van. (2006). Sexual and reproductive health: A matter of life and death. Lancet, 368(9547), 1595-1607. doi: https:// doi.org/10.1016/s0140-6736(06)69478-6.

Grond, E. (2011). Sexualität im Alter: Was Pflegekräfte wissen sollten und was sie tun können (2. Aufl.). Hannover: Schlütersche Verlagsgesellschaft.

Gupta, K. (2011). "Screw Health": Representations of sex as a health-promoting activity in medical and popular literature. Journal of Medical Humanities, 32(2), 127-140. doi: https://doi. org/10.1007/s10912-010-9129-x.

Jones, L., Bellis, M. A., Wood, S., Hughes, K., McCoy, E., Eckley, L., Bates, G., Mikton, C., Shakespeare, T., \& Officer, A. (2012). Prevalence and risk of violence against children with disabilities: A systematic review and meta-analysis of observational studies. Lancet, 380(9845), 899-907. doi: https://doi.org/10.1016/s0140-6736(12)60692-8. 
Levin, R. J. (2007). Sexual activity, health and well-being - The beneficial roles of coitus and masturbation. Sexual and Relationship Therapy, 22, 135-148. doi: https://doi. org/10.1080/14681990601149197.

Levy, D. (2007). Love and sex with robots: The evolution of human-robot relationships. New York: Harper Collins.

Lindsay, W. R. (2002). Research and literature on sex offenders with intellectual and developmental disabilities. Journal of Intellectual Disability Research, 46(1), 74-85. doi: https://doi. org/10.1046/j.1365-2788.2002.00006.x.

Liu, H., Waite, L. J., Shen, S., \& Wang, D. H. (2016). Is sex good for your health? A national study on partnered sexuality and cardiovascular risk among older men and women. Journal of Health and Social Behavior, 57(3), 276-296. doi: https://doi.org/10.1177/0022146516661597.

Lottes, I. L. (2013) Sexual rights: Meanings, controversies, and sexual health promotion. Journal of Sex Research, 50(3-4), 367-391. doi: https://doi.org/10.1080/00224499.2013.764380.

Lynae, E. (2016). Sex toys \& disabilities guide. http://erikalynae.com/2016/10/21/sex-toys-disability/. Zugegriffen: 1. März 2018.

Mahieu, L., \& Gastmans, C. (2015). Older residents' perspectives on aged sexuality in institutionalized elderly care: A systematic literature review. International Journal of Nursing Studies, 52(12), 1891-1905. doi: https://doi.org/10.1016/j.ijnurstu.2015.07.007.

Mahieu, L., Van Elssen, K., \& Gastmans, C. (2011). Nurses' perceptions of sexuality in institutionalized elderly: A literature review. International Journal of Nursing Studies, 48(9), 11401154. doi: https://doi.org/10.1016/j.ijnurstu.2011.05.013.

Marquardt, K. (2017). Ein Altenheim für homosexuelle Bewohner - Pflege ohne Diskriminierung. Heilberufe, 69(3), 40-42.

Matthias, R. E., Lubben, J. E., Atchison, K. A., \& Schweitzer, S. O. (1997). Sexual activity and satisfaction among very old adults: Results from a community-dwelling medicare population survey. Gerontologist, 37(1), 6-14. doi: https://doi.org/10.1093/geront/37.1.6.

Mattner, U. (2015). Ich will nicht nur kuscheln. Aktion Mensch. https://www.aktion-mensch.de/ leichte-sprache/magazin/gesellschaft_leichte_sprache/Rotlicht.html. Zugegriffen: 1. März 2018.

McCabe, M. P., \& Taleporos, G. (2003). Sexual esteem, sexual satisfaction, and sexual behavior among people with physical disability. Archives of Sexual Behavior, 32(4), 359-369.

Mück, H. (2013). Sexuelle Bedürfnisse, Paarbeziehungen und Sexualleben demenzkranker Menschen. pro familia Magazin 4, 16-19. https://www.profamilia.de/fileadmin/publikationen/Magazin/2013/pfm_4_2013.pdf. Zugegriffen: 1. März 2018.

Owens, T. (2014). Disability and sex: Let's be frank about sex toys. Disability horizonts. http:// disabilityhorizons.com/2014/07/disability-and-sex-lets-be-frank-about-sex-toys/. Zugegriffen: 1. März 2018.

Owens, T. (2015). Supporting disabled people with their sexual lives: A clear guide for health and social care professionals. London: Jessica Kingsley Publishers.

Palacios-Ceña, D., Martínez-Piedrola, R. M., Pérez-de-Heredia, M., Huertas-Hoyas, E., Carrasco-Garrido, P., \& Fernández-de-las-Peñas, C. (2016). Expressing sexuality in nursing homes. The experience of older women: A qualitative study. Geriatric Nursing, 37(6), 470-477. doi: https://doi.org/10.1016/j.gerinurse.2016.06.020.

Pearson, I. (2016). The future of sex report: The rise of the robosexuals. http://graphics.bondara. com/Future_sex_report.pdf. Zugegriffen: 1. März 2018.

Plummer, S. B., \& Findley, P. A. (2012). Women with disabilities' experience with physical and sexual abuse: Review of the literature and implications for the field. Trauma, Violence, \& Abuse, 13(1), 15-29. doi: https://doi.org/10.1177/1524838011426014.

Pro familia. (2005). Sexuelle Assistenz für Frauen und Männer mit Behinderungen. Expertise. https://www.profamilia.de/fileadmin/publikationen/Fachpublikationen/expertise_sexuelle_ assistenz.pdf. Zugegriffen: 1. März 2018. 
Reichert, M., \& Karrasch, R. M. (2013). Partnerschaftliche Pflegebeziehungen und Sexualität. Pro familia Magazin, 4, 10-13. https://www.profamilia.de/fileadmin/publikationen/Magazin/2013/ pfm_4_2013.pdf. Zugegriffen: 1. März 2018.

Robbins, C. L., Schick, V., \& Reece, M. (2011). Prevalence, frequency, and associations of masturbation with partnered sexual behaviors among US adolescents. Archives of Pediatrics \& Adolescent Medicine, 165(12), 1087-1093. doi: https://doi.org/10.1001/archpediatrics.2011.142.

Sandfort, L. (2010). Recht auf Liebeskummer - Emanzipatorische Sexualberatung für Behinderte. Neu-Ulm: AG SPAK Bücher.

Senf, G. (2013). Sexuelle Assistenz - Ein kontrovers diskutiertes Konzept. Psychother Dialog, 14(2), 68-71. doi: https://doi.org/10.1055/s-0033-1348370.

Shakespeare, T. (2000). Disabled sexuality: Toward rights and recognition. Sexuality and Disability, 18(3), 159-166.

Shakespeare, T., Gillespie-Sells, K., \& Davies, D. (1997). The sexual politics of diability: Untold Desires. London: Cassell.

Sharkey, N., Wynsberghe, A. van, Robbins, S., \& Hancock, E. (2017). Our sexual future with robots. Consultation report. Foundation for responsible robotics. https://responsible-robotics-myxf6pn3xr.netdna-ssl.com/wp-content/uploads/2017/11/FRR-Consultation-Report-Our-Sexual-Future-with-robots-1-1.pdf. Zugegriffen: 1. März 2018.

Siciliano, B., \& Khatib, O. (2016). Handbook of robotics (2. Aufl.). Heidelberg: Springer.

Sramek, G. (2015). Recht auf Sexualität. Beispiele aus der Praxis. In M. Kojer \& M. Schmidl (Hrsg.), Demenz und Palliative Geriatrie in der Praxis (S. 183-197). Heidelberg: Springer.

Tepper, M. S. (2000). Sexuality and disability: The missing discourse of pleasure. Sexuality and Disability, 18(4), 283-290.

Tiefer, L. (2002). The emerging global discourse of sexual rights. Journal of Sex \& Marital Therapy, 28(5), 439-444.

Tilley, E., Walmsley, J., Earle, S., \& Atkinson, D. (2012). ,The silence is roaring': Sterilization, reproductive rights and women with intellectual disabilities. Disability \& Society, 27(3), 413426. doi: https://doi.org/10.1080/09687599.2012.654991.

Vandemeulebroucke, T., Casterlé, B. D. de, \& Gastmans, C. (2017). How do older adults experience and perceive socially assistive robots in aged care: A systematic review of qualitative evidence. Aging \& Mental health, 22(2), 149-167. doi: https://doi.org/10.1080/13607863.2017 .1286455 .

Van der Vight-Klußmann, R. (2014). (Kein) Sex im Altenheim? Körperlichkeit und Sexualität in der Altenhilfe. Hannover: Schlütersche Verlagsgesellschaft.

Wacker, J., Macy, R., Barger, E., \& Parish, S. (2009). Sexual assault prevention for women with intellectual disabilities: a critical review of the evidence. Intellectual and Developmental Disabilities, 47(4), 249-262. doi: https://doi.org/10.1352/1934-9556-47.4.249.

Wilson, R. J., \& Burns, M. (2011). Intellectual disability and problems in sexual behaviour: Assessment, treatment, and promotion of healthy sexuality. Massachusetts: NEARI Press.

World Association for Sexual Health - WAS. (2014). Declaration of sexual rights. http://www. worldsexology.org/resources/declaration-of-sexual-rights/. Zugegriffen: DATUM.

World Health Organization - WHO. (1946). Constitution of the world health organization. http:// www.who.int/about/mission/en/. Zugegriffen: 1. März 2018.

World Health Organization - WHO. (2006). Defining sexual health. Consultation Report. http:// www.who.int/reproductivehealth/publications/sexual_health/defining_sh/en/. Zugegriffen: 1. März 2018.

Zinsmeister, J. (2017). Hat der Staat den Bürger*innen Sexualität zu ermöglichen? In U. Lembke (Hrsg.), Regulierungen des Intimen (S. 71-93). Wiesbaden: Springer. 
Prof. Dr. Nicola Döring, Diplom-Psychologin, Universitätsprofessorin für Medienpsychologie und Medienkonzeption an der Technischen Universität Ilmenau. Zu ihren Arbeitsschwerpunkten gehören psychische und soziale Aspekte der Online-, Mobil- und Mensch-Roboter-Kommunikation, Gender- und Sexualforschung sowie Forschungsmethoden und Evaluation. Sie ist Mitherausgeberin der Zeitschrift für Sexualforschung (Thieme Verlag). Web: www.nicola-doering.de.

Open Access Dieses Kapitel wird unter der Creative Commons Namensnennung 4.0 International Lizenz (http://creativecommons.org/licenses/by/4.0/deed.de) veröffentlicht, welche die Nutzung, Vervielfältigung, Bearbeitung, Verbreitung und Wiedergabe in jeglichem Medium und Format erlaubt, sofern Sie den/die ursprünglichen Autor(en) und die Quelle ordnungsgemäß nennen, einen Link zur Creative Commons Lizenz beifügen und angeben, ob Änderungen vorgenommen wurden.

Die in diesem Kapitel enthaltenen Bilder und sonstiges Drittmaterial unterliegen ebenfalls der genannten Creative Commons Lizenz, sofern sich aus der Abbildungslegende nichts anderes ergibt. Sofern das betreffende Material nicht unter der genannten Creative Commons Lizenz steht und die betreffende Handlung nicht nach gesetzlichen Vorschriften erlaubt ist, ist für die oben aufgeführten Weiterverwendungen des Materials die Einwilligung des jeweiligen Rechteinhabers einzuholen.

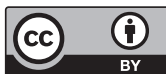

